\title{
Dephasing in Quantum Dots: Quadratic Coupling to Acoustic Phonons
}

\author{
E. A. Muljarov ${ }^{1,2, *}$ and R. Zimmermann ${ }^{1}$ \\ ${ }^{1}$ Institut für Physik der Humboldt-Universität zu Berlin, Newtonstrasse 15, D-12489 Berlin, Germany \\ ${ }^{2}$ General Physics Institute, Russian Academy of Sciences, Vavilova 38, Moscow 119991, Russia
}

(Received 19 December 2003; published 29 November 2004)

A microscopic theory of optical transitions in quantum dots with a carrier-phonon interaction is developed. Virtual transitions into higher confined states with acoustic phonon assistance add a quadratic phonon coupling to the standard linear one, thus extending the independent boson model. Summing infinitely many diagrams in the cumulant, a numerically exact solution for the interband polarization is found. Its full time dependence and the absorption line shape of the quantum dot are calculated. It is the quadratic interaction which gives rise to a temperature-dependent broadening of the zero-phonon line, calculated here for the first time in a consistent scheme.

PACS numbers: 78.67.Hc, 71.38.-k

The electron-lattice interaction in quantum dots (QDs) plays a decisive role for understanding the time evolution and dephasing mechanisms after optical excitation. Highresolution photoluminescence spectra on single $\mathrm{CdZnTe}$ QDs show a relatively narrow zero-phonon line (ZPL) with Lorentz broadening $\Gamma$ depending on temperature on top of a broadband [1]. The latter is a superposition of acoustic phonon satellites. Complementary and more detailed information on the polarization dynamics and dephasing has been obtained from four-wave mixing measurements in an ensemble of InGaAs QDs [2]. The polarization decays initially very fast during a few picoseconds (formation of the broadband), followed by a much longer exponential decay (tens to hundreds of picoseconds) reflecting the ZPL broadening. This scenario is referred to in the literature as pure dephasing [3].

In theory, the working horse has been the independent boson model [4] which allows an exact analytical solution for the linear optical polarization [1,5-7] and for the photon echo [8] of QDs. This model starts with a carrier-phonon Hamiltonian being linear in the phonon displacement operators, and diagonal in the confined electronic states. The exact solution employs the cumulant method and satisfactorily describes the broad phonon band, i.e., the initial decay of the polarization. However, the ZPL shows no broadening at all (no long-time decay), since the phonon cloud induced by the impulsive excitation of the QD relaxes quickly to a finite lattice distortion which stays constant during the subsequent time evolution. To simulate the (experimentally observed) ZPL broadening, a polarization decay can be introduced by hand $[1,6,8]$, or a phonon damping can be added phenomenologically via the Grüneisen effect [7].

To improve on this unsatisfying situation, we develop a purely microscopic approach to the dephasing in QDs which contains a finite broadening of the ZPL, thus going essentially beyond the independent boson model. Starting with the Hamiltonian $H=H_{0}+H_{\mathrm{d}}+H_{\text {nd }}$ we take into account the nondiagonal terms $H_{\text {nd }}$ of the carrier-phonon interaction, i.e., phonon-assisted transitions of electrons and holes to higher QD levels [3]. As the acoustic phonons which couple to the QD have energies not more than a few meV, these transitions are of virtual character only, and do not change the carrier occupation. In order to eliminate the off-diagonal part in first order, we apply a unitary transformation [9] $H^{\prime}=e^{S} H e^{-S} \approx H+[S, H]+$ $1 / 2[S,[S, H]]$ and choose $\left[S, H_{0}\right]=-H_{\mathrm{nd}}$. As a consequence, a term quadratic in the phonon displacement appears. However, $H^{\prime}$ allows still a separation of the electron and phonon coordinates as in the independent boson model, and we present an exact solution of this problem for the first time.

With optical phonons, such a quadratic coupling in QDs via virtual sublevel transitions has been introduced recently by Uskov et al. [10] and treated up to second order in the cumulant. The quadratic Hamiltonian $H^{\prime}$ is valid only if the typical phonon energy is much smaller than a characteristic energy distance between exciton levels. However, this condition is not fulfilled for optical phonons in InGaAs QDs [10]. At the same time the use of a finite phonon damping [10] makes the quadratic coupling completely irrelevant. In fact, as is shown in Ref. [7], with such a phenomenological parameter, the theory gives a finite ZPL broadening even without any virtual or real transitions to higher states. Finally, if phonon damping is absent, the neglect of the optical phonon dispersion as done in Ref. [10] leads to a Gauss decay of the polarization. In contrast, earlier theoretical works [11-13] on impurity-phonon interaction resulted in an exponential decay, $\exp (-\Gamma t)$, in agreement with the experiment.

The quadratic coupling with acoustic phonons for the ZPL decay was also explored recently by Goupalov et al. [14] and Hizhnyakov et al. [15] but assumed to be diagonal in phonon momentum. This is not correct since the QD breaks the translational symmetry. All these approaches [11-15], however, make use of a long-time expansion and are not able to describe the initial rapid decay (the broadband).

After eliminating the intersublevel coupling in first order we obtain [16] 


$$
\begin{gathered}
H^{\prime}=\sum_{\mathbf{q}} \hbar \omega_{\mathbf{q}} a_{\mathbf{q}}^{\dagger} a_{\mathbf{q}}+\left(\hbar \omega_{e h}+V\right)|1\rangle\langle 1|, \\
V=\sum_{\mathbf{q}} M_{\mathbf{q}}\left(a_{\mathbf{q}}+a_{-\mathbf{q}}^{\dagger}\right)-\sum_{\mathbf{q} \mathbf{q}^{\prime}} Q_{\mathbf{q} \mathbf{q}^{\prime}}\left(a_{\mathbf{q}}+a_{-\mathbf{q}}^{\dagger}\right)\left(a_{\mathbf{q}^{\prime}}+a_{-\mathbf{q}^{\prime}}^{\dagger}\right), \\
M_{\mathbf{q}}=M_{\mathbf{q} e}^{11}-M_{\mathbf{q} h}^{11}, \quad Q_{\mathbf{q} \mathbf{q}^{\prime}}=\sum_{a=e, h} \sum_{\nu \neq 1} \frac{M_{\mathbf{q} a}^{1 \nu} M^{\nu} \mathbf{q}^{\prime} a}{E_{\nu}^{a}-E_{1}^{a}} .
\end{gathered}
$$

The electronic Hilbert space consists of the QD ground state $|0\rangle$ and the excited-state $|1\rangle$ having just one electron in the lowest conduction-band level $\left(E_{1}^{e}\right)$ and one hole in the uppermost valence-band level $\left(E_{1}^{h}\right)$. The bare transition energy is denoted by $\hbar \omega_{e h}=E_{g}+E_{1}^{e}+E_{1}^{h}$. The matrix element for the deformation potential coupling with longitudinal acoustic phonons in the QD reads $(a=e, h)$

$$
M_{\mathbf{q} a}^{\nu \nu^{\prime}}=\sqrt{\frac{\hbar \omega_{q}}{2 \rho_{M} u_{s}^{2} V}} D_{a} \int d \mathbf{r} \psi_{\nu a}^{*}(\mathbf{r}) e^{i \mathbf{q r}} \psi_{\nu^{\prime} a}(\mathbf{r}),
$$

with $\psi_{\nu a}(\mathbf{r})$ and $D_{a}$ being the confinement wave function and the deformation potential constant, respectively. $\rho_{M}$ is the mass density, $u_{s}$ the sound velocity, and $\mathcal{V}$ the phonon normalization volume. In writing Eq. (4) we have neglected (i) the excitonic Coulomb correlation which is of minor importance for small QDs [7] but could be included easily; (ii) any difference between phonon parameters in the QD and in the barrier material leading to confined modes, thus dealing with bulk phonons only; (iii) deviations of the acoustic phonon dispersion from a linear isotropic one, $\omega_{\mathbf{q}}=u_{s} q$.

The linear response function, i.e., the polarization after a delta-pulse excitation at $t=0$, is given by the dipole-dipole correlation function $P(t)=i\left\langle d^{\dagger}(t) d(0)\right\rangle$ for $t>0$,

$$
\begin{aligned}
P(t)= & i e^{-i \omega_{e h} t}\left\langle\mathcal{T} \exp \left[-\frac{i}{\hbar} \int_{0}^{t} d \tau V(\tau)\right]\right\rangle \\
= & i e^{-i \omega_{e h} t} \exp \left[\sum_{n=1}^{\infty}\left(\frac{-i}{\hbar}\right)^{n} \frac{1}{n !} \int_{0}^{t} d t_{1} \ldots d t_{n}\right. \\
& \left.\times\left\langle\mathcal{T} V\left(t_{1}\right) \ldots V\left(t_{n}\right)\right\rangle_{\text {conn }}\right]
\end{aligned}
$$

with $\mathcal{T}$ being the time ordering, and using the interaction representation of operators. The expectation value for the electronic system is already performed, leaving only the average over the phonon operators to be done. In the second line of Eq. (5) we have used the cumulant expansion [4] which has only connected diagrams in the exponent. Applying Wick's theorem, the thermal phonon average forces the displacement operators to appear only pairwise in expectation values, which defines the (momentum diagonal) phonon propagator

$$
\begin{aligned}
D_{\mathbf{q}}(t) & =(-i / \hbar)\left\langle\mathcal{T}\left[a_{\mathbf{q}}(t)+a_{-\mathbf{q}}^{\dagger}(t)\right]^{\dagger}\left[a_{\mathbf{q}}(0)+a_{-\mathbf{q}}^{\dagger}(0)\right]\right\rangle \\
& =(-i / \hbar)\left[\left(N_{\mathbf{q}}+1\right) e^{-i \omega_{\mathbf{q}}|t|}+N_{\mathbf{q}} e^{i \omega_{\mathbf{q}}|t|}\right]
\end{aligned}
$$

as building block. The phonon occupation enters via the Bose function, $N_{\mathbf{q}}=1 /\left[\exp \left(\hbar \omega_{\mathbf{q}} / k_{B} T\right)-1\right]$. In the cumulant technique, connected diagrams are those which do not factorize. Thus, the propagators have to appear in different time integrations, forming a chainlike structure. Concentrating first on the linear interaction, there is only one diagram which is connected [ $n=2$ in Eq. (5)]. In all higher orders, the phonon propagators have their time integrations exclusively, and the expressions do factorize. Things are quite different for the quadratic interaction since now two displacement operators share the same time argument (but have different momentum). To form a connected diagram, they have to be distributed on two different propagators, ending up with a closed loop. Finally, a combination of linear and quadratic interactions adds another connected structure, which is a chain with two open ends. The linear coupling can appear only twice, just at the ends.

The calculation method for the diagram series developed in this work can be applied to any well-behaved function $Q_{\mathbf{q q}^{\prime}}$. A technical simplification can be achieved if a simple factorization of the dependence on $\mathbf{q}$ and $\mathbf{q}^{\prime}$ holds. Physically, this case may appear if one excited level having the smallest energy distance dominates the sum in Eq. (3). Concentrating at the moment on the next hole level $E_{2}^{h}$, we have with $\Delta_{h}=E_{2}^{h}-E_{1}^{h}$ the product form $Q_{\mathbf{q} \mathbf{q}^{\prime}}=M_{\mathbf{q} h}^{12} M_{\mathbf{q}^{\prime} h}^{21} / \Delta_{h}$. Then, summing over momentum, only three different propagators appear,

$$
\begin{aligned}
& D_{L}(t)=1 / \hbar \sum_{\mathbf{q}}\left|M_{\mathbf{q}}\right|^{2} D_{\mathbf{q}}(t), \\
& D_{Q}(t)=-2 / \Delta_{h} \sum_{\mathbf{q}}\left|M_{\mathbf{q} h}^{12}\right|^{2} D_{\mathbf{q}}(t), \\
& D_{M}(t)=\sqrt{2 /\left(\hbar \Delta_{h}\right)} \sum_{\mathbf{q}} M_{\mathbf{q}}^{*} M_{\mathbf{q} h}^{12} D_{\mathbf{q}}(t) .
\end{aligned}
$$

As a result we obtain the cumulant expansion

$$
P(t)=i \exp \left[-i \omega_{e h} t+K_{L}(t)+K_{Q}(t)+K_{M}(t)\right]
$$

with

$$
\begin{aligned}
K_{L}(t)= & -\frac{i}{2} \int_{0}^{t} d t_{1} d t_{2} D_{L}\left(t_{1}-t_{2}\right) \\
K_{Q}(t)= & \frac{1}{2} \sum_{n=1}^{\infty} \frac{1}{n} \int_{0}^{t} d t_{1} \ldots d t_{n} D_{Q}\left(t_{1}-t_{2}\right) \\
& \times D_{Q}\left(t_{2}-t_{3}\right) \ldots D_{Q}\left(t_{n}-t_{1}\right) \\
K_{M}(t)= & \frac{i}{2} \sum_{n=1}^{\infty} \int_{0}^{t} d t_{0} \ldots d t_{n+1} D_{M}\left(t_{0}-t_{1}\right) \\
& \times D_{Q}\left(t_{1}-t_{2}\right) \ldots D_{Q}\left(t_{n-1}-t_{n}\right) D_{M}\left(t_{n}-t_{n+1}\right)
\end{aligned}
$$

The first term in Eq. (9), $K_{L}$, is all what remains from the 
linear coupling, since only one diagram survives as discussed above. This reproduces exactly the result within the independent boson model. The quadratic interaction brings in a purely quadratic term, $K_{Q}$, and a mixed one, $K_{M}$, which both contain an infinite sum over diagrams. Note that the factorial in Eq. (5) is reduced to $1 / n$ and 1, respectively, due to a proper counting of equivalent diagrams.

For realistic coupling strengths, a calculation of the sum over $n$ term by term is hopeless. We follow another path which allows us to sum up the infinite sum numerically exact: With the quadratic propagator $D_{Q}(t)$ as kernel, we look for the eigenvalue problem of the Fredholm integral equation

$$
\int_{0}^{t} d t_{2} D_{Q}\left(t_{1}-t_{2}\right) u_{j}\left(t_{2}\right)=\Lambda_{j} u_{j}\left(t_{1}\right) .
$$

The kernel is complex and symmetric, but not Hermitian. Therefore, the eigenvalues $\Lambda_{j}(j=1,2, \cdots)$ are complex, but the eigenfunctions still form an orthonormal system. Expanding the multiple integrals into the orthonormal system, an $n$-fold convolution of $D_{Q}(t)$ converts into a power $\Lambda_{j}^{n}$. The subsequent summation over $n$ results in

$$
\begin{gathered}
K_{Q}(t)=-\frac{1}{2} \sum_{j} \ln \left(1-\Lambda_{j}\right), \\
K_{M}(t)=\frac{i}{2} \sum_{j} \frac{D_{M j}^{2}}{1-\Lambda_{j}},
\end{gathered}
$$

where

$$
D_{M j}=\int_{0}^{t} d t_{1} d t_{2} D_{M}\left(t_{1}-t_{2}\right) u_{j}\left(t_{2}\right) .
$$

Technically, we have discretized the time integrations on a fine grid, thus converting the Fredholm problem to a standard matrix eigenvalue search.

Apart from the first excited hole level only the factorization of $Q_{\mathbf{q q}^{\prime}}$ can be employed for several excited levels if they refer to different spatial symmetries. E.g., if the quantum dot has mirror symmetry, several excited odd states of different orientation have vanishing cross terms in the quadratic cumulant $D_{Q}(t)$, and contribute additively to $K_{Q}(t)$. According to the same argument, $D_{M}(t) \equiv 0$ and consequently $K_{M}(t) \equiv 0$. In the actual calculation we assume a spherical quantum dot with parabolic confinement potential. The wave functions of the lowest states, $\psi_{1 a}(\mathbf{r})$, are isotropic Gauss orbitals with variance $l_{a}$. Taking artificially $l_{e}=l_{h}=l$ allows us to take into account the next three (degenerate) excited states for both, electron and hole simultaneously, without spoiling the factorization scheme. The only changes are the replacement $D_{v}^{2} / \Delta_{h} \rightarrow D_{c}^{2} / \Delta_{e}+D_{v}^{2} / \Delta_{h}$ in the definition of $D_{Q}(t)$, and an additional factor of 3 in $K_{Q}(t)$, Eq. (11). In the general case of a nonfactorizable $Q_{\mathbf{q q}}$ one should treat $D_{Q}(t)$ as a matrix and $D_{M}(t)$ as a vector in momentum space.
In the calculations, we have taken InAs parameters [17] $\rho_{M}=5.67 \mathrm{~g} / \mathrm{cm}^{3}, \quad u_{s}=4.6 \times 10^{3} \mathrm{~m} / \mathrm{s}, \quad D_{c}=$ $-13.6 \mathrm{eV}, D_{c}-D_{v}=-6.5 \mathrm{eV}$. The confinement variance $l$ is the only parameter characterizing the QD. The matrix element Eq. (4) behaves like $\left(M_{\mathbf{q} a}^{11}\right)^{2} \propto$ $q \exp \left[-(q l)^{2} / 2\right]$, which gives for the different propagators a temporal decay on the scale $l / u_{s}$. Consequently, $\hbar u_{s} / l$ sets the scale for the phonon energies involved, and gives roughly the spectral width of the phonon satellites (broadband). For the QDs studied in Ref. [2], we have extracted a value of $l \approx 3.3 \mathrm{~nm}$. To come closer to the experimental situation of a more pancake shaped quantum dot, we have taken the experimental observation of $\Delta_{e}+\Delta_{h}=65 \mathrm{meV}$ and distributed between electron and hole according to the InAs mass ratio of 10:1.

The calculated polarization amplitude in Fig. 1 undergoes a quick initial decay on the scale $l / u_{s} \approx 1 \mathrm{ps,} \mathrm{which}$ reflects the formation of the polaron cloud around the $e-h$ pair. This state, however, is not completely stable: Because of the quadratic coupling, there are virtual scattering events into higher states which distort the polarization, leading to an exponential decay (pure dephasing). Note that the quadratic coupling does not change much the initial decay, as seen from the dashed curve in Fig. 1 where a decay is added by hand to the linear result.

For times much larger than the initial decay $\left(\propto l / u_{s}\right)$ a more detailed analysis is possible. The linear term alone gives $K_{L}(t) \rightarrow-S_{L}+i \Omega_{L} t$, which is a reduction of the polarization amplitude $\exp (-S)$ ( $S$ is the Huang-Rhys factor) and a purely imaginary contribution linear in time (polaron shift $\Omega$ ). The quadratic term can be analyzed in terms of the eigenvalues $\Lambda_{j}$. For $t$ larger than the temporal width of $D_{Q}\left(t_{1}\right)$, the eigenfunctions are found to

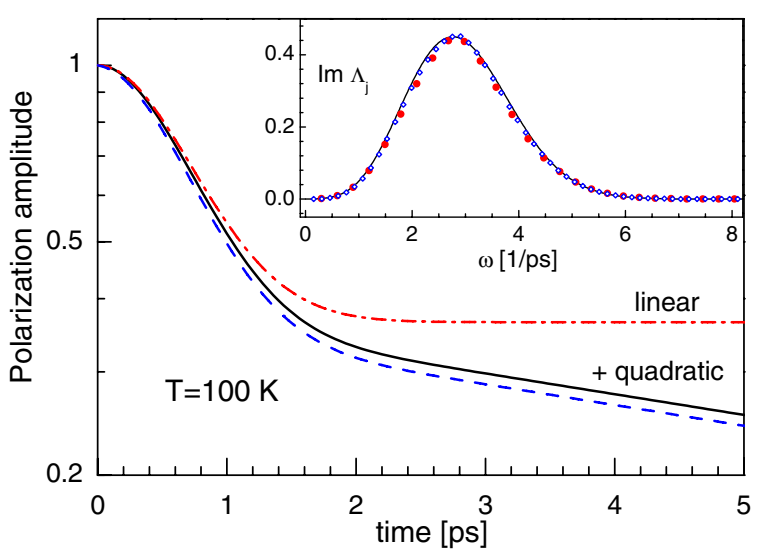

FIG. 1 (color online). Polarization amplitude calculated for an InAs QD with $l=3.3 \mathrm{~nm}$ at $T=100 \mathrm{~K}$ with linear and quadratic coupling to acoustic phonons. For the dashed curve, a finite decay $1 / \Gamma=12.3 \mathrm{ps}$ has been added by hand to the linear result. The inset shows the (dominant) imaginary part of the eigenvalues $\Lambda_{j}$ at $\omega_{j}$ for $t=10 \mathrm{ps}$ (circles) and $20 \mathrm{ps}$ (diamonds), superimposed to the Fourier transform $\tilde{D}_{Q}(\omega)$ (solid curve). 


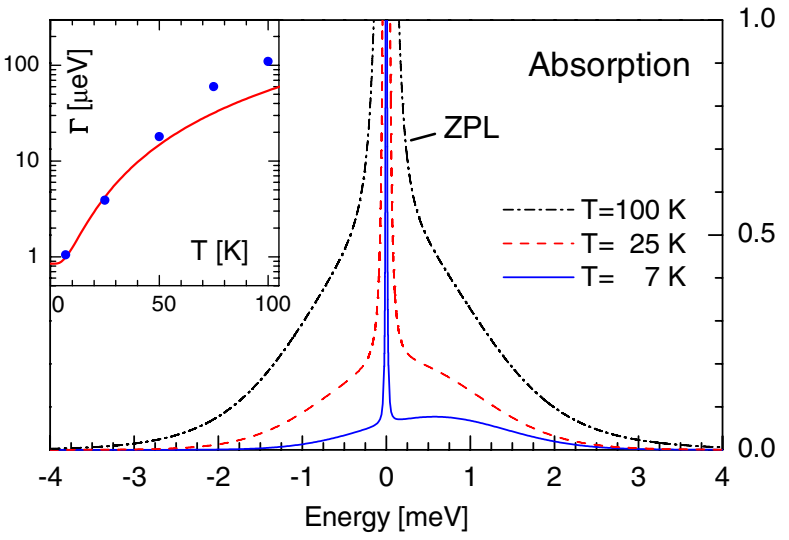

FIG. 2 (color online). Absorption spectra of an InAs QD with carrier confinement length of $l=3.3 \mathrm{~nm}$ at different temperatures. The ZPL transition energy is taken as zero of energy. Inset: Calculated broadening of the ZPL compared with the experimental results by Borri et al. [2] (circles). A radiative rate of $0.85 \mu \mathrm{eV}$ is added to the calculated phonon-mediated decay rate.

oscillate in the middle of the interval as $u_{j}\left(t_{1}\right) \propto$ $\exp \left(i \omega_{j} t_{1}\right)$, while $\Lambda_{j}=\tilde{D}_{Q}\left(\omega_{j}\right)$ with the Fourier transform of $D_{Q}\left(t_{1}\right)$. The exact position of the frequencies $\omega_{j}$ is determined by the boundary conditions at $t_{1}=0$ and $t_{1}=t$. Numerically, we found very good agreement with

$$
\Lambda_{j}(t) \approx \tilde{D}_{Q}\left(\omega_{j}=\frac{\pi j}{t+2 \tau}\right) .
$$

In the inset of Fig. 1 we plot the eigenvalues at two different times, adjusting $\tau=0.27$ ps for optimal agreement with $\tilde{D}_{Q}(\omega)$ (solid curve). Notice that for $t=20 \mathrm{ps}$ the eigenvalues are 2 times denser than for $t=10 \mathrm{ps}$, as dictated by Eq. (13). Combining Eqs. (11) and (13) we arrive at

$$
\begin{aligned}
K_{Q}(t) & \rightarrow-(t+2 \tau) \int_{0}^{\infty} \frac{d \omega}{2 \pi} \ln \left[1-\tilde{D}_{Q}(\omega)\right] \\
& =-S_{Q}-\Gamma t+i \Omega_{Q} t,
\end{aligned}
$$

which has now a real part in the linear time dependence. This gives the polarization dephasing or ZPL width in the frequency domain. These analytical expressions for dephasing rate $\Gamma$ and frequency shift $\Omega_{Q}$ have been derived earlier in different models of the quadratic electronphonon coupling [11-15]. New is the inclusion of the time-independent correction $\propto \tau$ which changes the Huang-Rhys factor $S=S_{L}+S_{Q}$. Since $S_{Q}$ is complex the ZPL Lorentzian gets a (small) dispersive contribution.

From the complex polarization follows the absorption spectrum easily, $\alpha(\omega)=\operatorname{Im} \int_{0}^{\infty} d t P(t) \exp (i \omega t)$ (Fig. 2). At low temperatures, the broadband is distinctly asymmetric and gets more symmetric as $T$ grows. This is not much different from the results of linear coupling. However, in accordance with the decay seen in Fig. 1, the ZPL acquires a Lorentz broadening with width $\Gamma(T)$, which is exclusively due to the quadratic coupling. The inset shows the calculated temperature dependence of the dephasing rate $\Gamma(T)$ (solid curve). The comparison with the experimental data (circles) by Borri et al. [2] shows that our theory without adjustable parameters gives the right magnitude of the ZPL broadening. Including virtual transitions to further higher-lying states would improve the agreement. This calls, however, for a proper description of states in the wetting layer which is beyond the scope of the present Letter.

In conclusion, we have developed a microscopic theory of dephasing in quantum dots accounting for quadratic coupling between carriers and acoustic phonons. Introducing a new numerical method allowed us to perform the infinite sum of diagrams in the cumulant. This exact solution gives the full time dependence of the optical polarization. The absorption spectrum includes the broadband and the broadening of the zero-phonon line on the same footing. Our calculated results for the ZPL decay in InGaAs quantum dots show the same trend and magnitudes as found in the experiment [2].

Financial support by DFG (Sfb 296), DAAD (NATO Grant No. 325-A/03/06772), and the Russian Foundation for Basic Research is gratefully acknowledged.

*Electronic address: muljarov@gpi.ru

[1] L. Besombes, K. Kheng, L. Marsal, and H. Mariette, Phys. Rev. B 63, 155307 (2001).

[2] P. Borri et al., Phys. Rev. Lett. 87, 157401 (2001).

[3] T. Takagahara, Phys. Rev. B 60, 2638 (1999).

[4] G. Mahan, Many-Particle Physics (Plenum, New York, 1990).

[5] S. Schmitt-Rink, D. A. B. Miller, and D. S. Chemla, Phys. Rev. B 35, 8113 (1987).

[6] B. Krummheuer, V. M. Axt, and T. Kuhn, Phys. Rev. B 65, 195313 (2002).

[7] R. Zimmermann and E. Runge, in Proceedings of the 26th ICPS, Edinburgh, Scotland, United Kingdom (Inst. of Physics Publ., Bristol and Philadelphia, 2002).

[8] A. Vagov, V. M. Axt, and T. Kuhn, Phys. Rev. B 66, 165312 (2002).

[9] The unitary transformation is similar to what is standard in quantum field theory, L. L. Foldy and S. A. Wouthuysen, Phys. Rev. 78, 29 (1950).

[10] A. V. Uskov et al., Phys. Rev. Lett. 85, 1516 (2000).

[11] G. F. Levenson, Phys. Status Solidi B 43, 739 (1971).

[12] I. S. Osad'ko, Fiz. Tverd. Tela 14, 2927 (1972) [Sov. Phys. Solid State 14, 2522 (1973)].

[13] D. Hsu and J. L. Skinner, J. Chem. Phys. 81, 1604 (1984).

[14] S. V. Goupalov, R. A. Suris, P. Lavallard, and D. Citrin, IEEE J. Sel. Top. Quantum Electron. 8, 1009 (2002).

[15] V. Hizhnyakov, H. Kaasik, and I. Sildos, Phys. Status Solidi B 234, 644 (2002).

[16] The excited-state Hamiltonian with quadratic phonon terms is stable under the present weak-couping conditions pertinent for the InAs QD. The small parameter is coupling strength $\left|M_{\mathbf{q} a}^{\nu 1}\right|$ over level distance $\left|E_{\nu}^{a}-E_{1}^{a}\right|$.

[17] Semiconductors, Landoldt-Börnstein New Series, edited by O. Madelung (Springer, Berlin, 1986), Vol. 22a. 